\title{
Functional Outcome of Chronic Anterior Cruciate Ligament Rupture Reconstruction Using Peroneus Longus Tendon Graft According to AOFAS and IKDC Score: A Case Series
}

\author{
Rizki Zainuraditya, I. Gusti Ngurah Wien Aryana
}

\section{ABSTRACT}

\begin{abstract}
Introduction: The anterior cruciate ligament (ACL) is a crucial ligament structure of the knee that plays a significant role in knee joint stability and competitive sports performance. In order to reestablish knee stability, ACL reconstruction (ACLR) in the presence of rupture has been recognized as the most common surgical management procedure.
\end{abstract}

Case: This study reported six cases of chronic ACL rupture reconstruction using peroneus longus tendon graft. All patients underwent an arthroscopic ACL repair procedure in Sanglah Hospital Bali. In terms of functional outcome, all patients were assessed using the scoring of AOFAS for the ankle and IKDC for the knee. Result: The functional outcomes of the IKDC and AOFAS score were good from all six patients who already underwent ACL reconstruction using peroneus longus graft with a minimum followup of one year.

Discussion: Chronic ACL ruptures were successfully treated using the peroneus longus tendon graft. Intraoperatively, there were no significant problems from the harvested peroneus longus tendon graft and ACL reconstruction with the graft. All patients showed no complications, including the local infection following the operation. Rehabilitation procedure post-ACL reconstruction was performed at the Rehabilitation Unit in Sanglah General Hospital.

Conclusion: Peroneus longus tendon graft is considered a suitable choice of graft for ACL reconstruction. It presented satisfactory results, effectiveness, and safety based on the AOFAS and IKDC scores.

Keywords: Anterior cruciate ligament, AOFAS, IKDC, Peroneus longus tendon graft, reconstruction.

\section{INTRODUCTION}

The anterior cruciate ligament (ACL) is a crucial ligament of the knee structure that plays a significant role in knee joint stability and competitive sports performance. Approximately 200,000 patients present with ACL injuries each year in the United States, and more than half of these patients underwent surgical repair or reconstruction. The ACL reconstruction (ACLR) has been recognized as the most common procedure to restore knee stability in the presence of rupture. However, nearly $4.0 \%$ to $15.3 \%$ of the surgeries ended up with unsatisfactory results. Previous literature declared that a series of factors could influence the outcomes of ACLR surgery, including age, sports activities, the type of graft, initial graft tension, graft diameter, and anatomical reconstruction [1], [2].
Submitted: August 6, 2021

Published: August 29, 2021

ISSN: 2593-8339

DOI: 10.24018 / ejmed.2021.3.4.1002

\section{R. Zainuraditya *}

Orthopaedic Resident, Department of Orthopaedic and Traumatology, Sanglah General Hospital/Faculty of Medicine, University of Udayana, Denpasar, Bali, Indonesia.

(e-mail: rizkiortho@gmail.com)

I. G. N. W. Aryana

Orthopaedic Consultant, Department of Orthopaedic and Traumatology, Sanglah General Hospital/Faculty of Medicine, University of Udayana, Denpasar, Bali, Indonesia.

*Corresponding Author
Anatomic ACLR has been considered a satisfactory surgical procedure in sports medicine, and the autograft has been commonly used for ACLR in the past. An autograft is related to earlier bone unification and tendon-bone healing, as well as reduced immunological rejection after transplantation. In addition, there is a lesser risk of disease transmission. In clinical practice, the semitendinosus tendongracilis (STG) autograft is typically harvested from the operated knee [2].

Nowadays, bone-patellar, tendon-bone, or double-looped semitendinosus/gracilis autografts have become the most common graft choices for ACLR. Other common sources of autograft include the fascia lata, iliotibial band, and quadriceps tendon with or without bone. The main disadvantages of Bone-Patellar-Bone are risks of patellar fracture, knee extension weakness, and anterior knee pain. In addition, in many situations, the hamstrings grafts fall short in diameter and length. The resulting post-operative 
hamstring weakness also makes the knee susceptible to an unprotected anterior drawer force. Thus, the clinical outcomes of ACL reconstruction using the peroneus longus tendon seemed satisfactory regarding the evaluation of knee function [3], [4].

This study presented a series of seven chronic ACL rupture cases, which were reconstructed using the peroneus longus tendon graft. All patients underwent an arthroscopic ACL repair procedure in Sanglah General Hospital, Bali. In terms of functional outcome, all patients were followed up using the American Orthopaedic Foot \& Ankle Society (AOFAS) for ankle and International Knee Documentation Committee (IKDC) for the knee.

\section{CASE PRESENTAtion}

\section{A. Case 1}

A 36-year-old female came to the orthopedic outpatient clinic in Sanglah General Hospital, complaining of chronic pain in her right knee in the last six months before admission (February 2017). The symptom began after the patient had a motorcycle accident and fell to the right side, bumping her right knee to the ground. The pain was intermittent and worsen whenever the patient walks upstairs or downstairs. The patient was also unable to run, and the pain was relieved by rest. The patient had no treatment before for this complaint (see Fig.1 and 2).

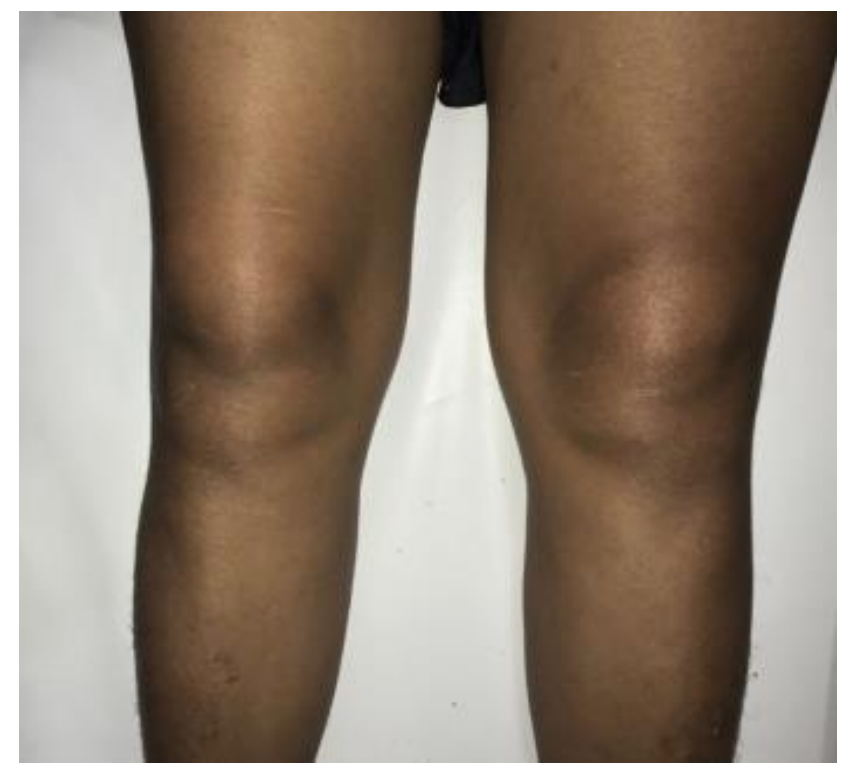

Fig. 1. Clinical features of the patient on the first case.

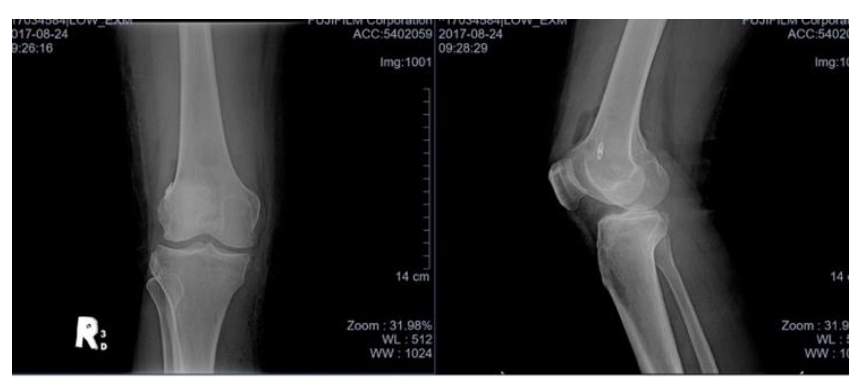

Fig. 2. Post-operative radiographic result of the patient on the first case.

From the physical examination, there was not any swelling deformity. Palpation showed no tenderness around the affected knee. The examination of capillary refill time, dorsalis pedis artery, and pulse oximetry were within normal limit. Positive results were found on Lachman Test and Anterior Drawer Test, but not significant on the Posterior Drawer Test. The patient was diagnosed with right anterior cruciate ligament rupture and was performed ACLR using the peroneus longus tendon graft in August 2017. The patient did not show any surgical complications during the follow-up.

\section{B. Case 2}

A 58-year-old male came to the orthopedic outpatient clinic in Sanglah General Hospital, complaining of pain in his left knee in the past six months prior to admission (March 2017). The complaint started when the patient accidentally twisted his left knee during exercise. Since the past month, the patient felt that the pain worsened, especially when walking upstairs or downstairs and during high-intensity activities (see Fig. 3 and 4).

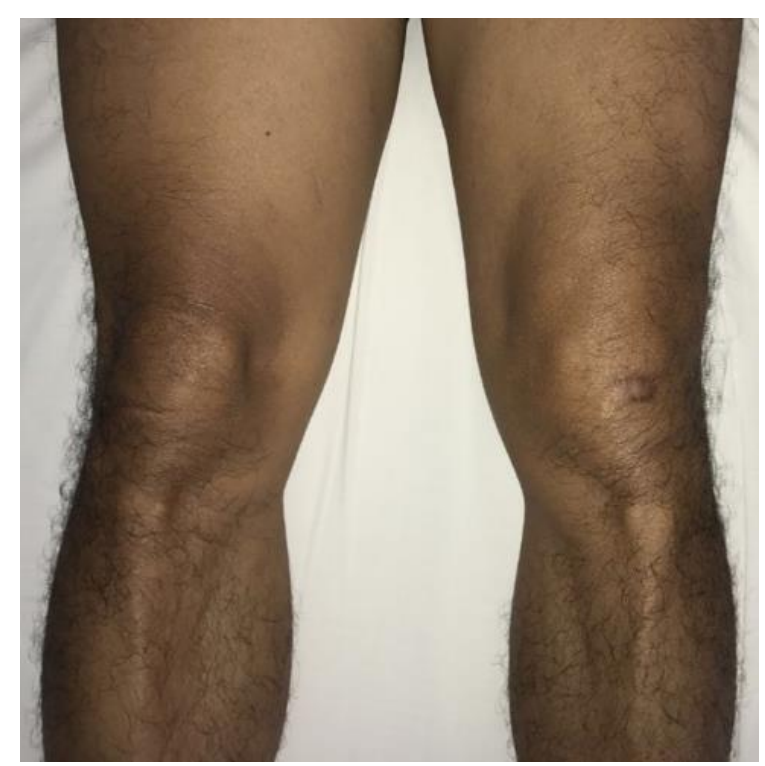

Fig. 3. Clinical features of the left ruptured ACL from the second case.

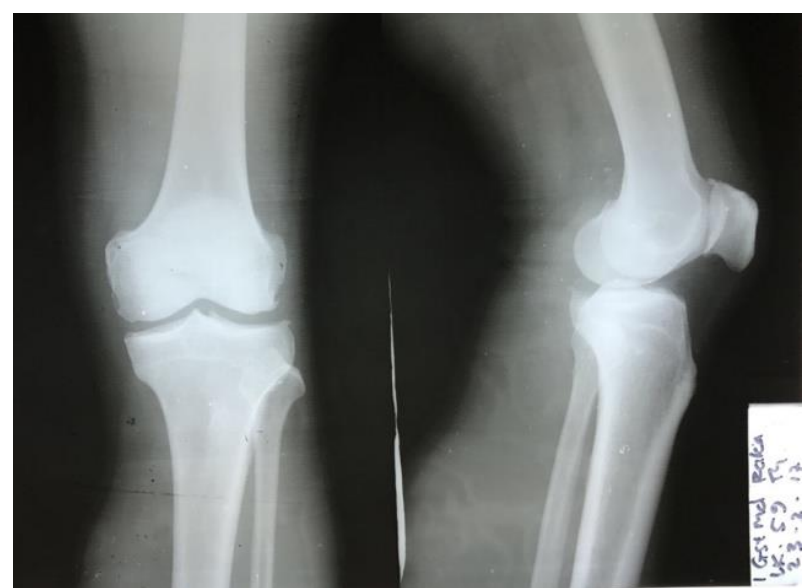

Fig. 4. Radiographic result of the patient on the second case.

Physical examination revealed no swelling, deformity, or pain on palpation. The capillary refill time, dorsalis pedis artery, and pulse oximetry were within normal limit. The Lachman Test and Anterior Drawer Test on the left knee revealed a positive result. The patient was diagnosed with left anterior cruciate ligament rupture and was performed ACLR using peroneus longus tendon graft in August 2017. The 
patient did not show any surgical complications during the follow-up.

\section{Case 3}

A 23-year-old male came to the orthopedic outpatient clinic in Sanglah General Hospital with an unstable sensation on his right knee. Two years before the symptoms began, the opponent kicked the patient on his right knee during exercise. The complaint became worse, mainly when the patient walks upstairs or downstairs and during high-intensity activity.

On physical examination, the patient presented with no swelling or deformity, as well as any tenderness around the affected knee. The capillary refill time, dorsalis pedis artery, and pulse oximetry examination were within normal limit. The Lachman Test and Anterior Drawer Test were found positive on the left knee. The Magnetic Resonance Imaging (MRI) examination on the affected knee confirmed the presence of a ruptured left anterior cruciate ligament (September 2016) (see Fig. 5 and 6). The patient underwent an ACLR procedure using the peroneus longus tendon graft in March 2017. The patient exhibited no further surgical complications during the follow-up.

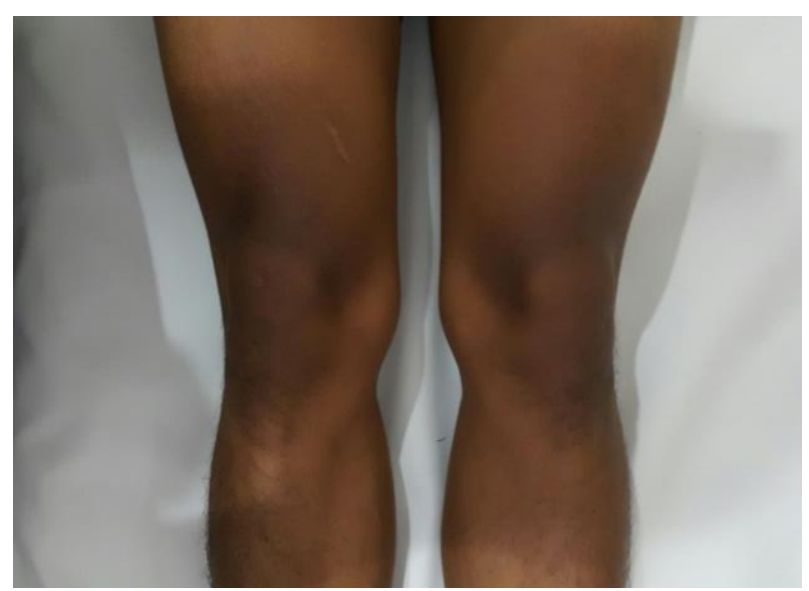

Fig. 5. Clinical features of the left ACL rupture from the third case.

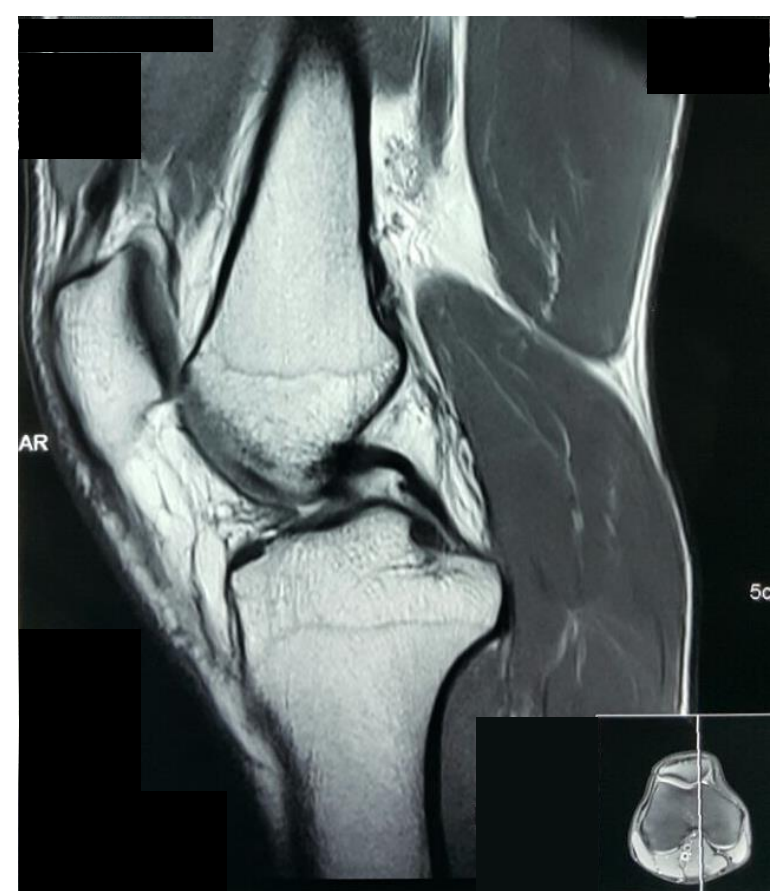

Fig. 6. Figure showing an MRI of ruptured ACL on the right knee.

\section{Case 4}

A 19-year-old male came to the orthopedic outpatient clinic in Sanglah General Hospital with a complaint of persistent pain on his left knee for the last one year prior to admission (2018). The patient reported a history of falling from the stairs, twisting his left knee then bumped to the floor. The patient continued having a worsening period of knee instability and was unable to do sports activities.

The physical examination showed no swelling, deformity, or tenderness around the left knee. The capillary refill time, dorsalis pedis artery, and pulse oximetry tests were within normal limit. The Lachman Test and Anterior Drawer Test were positive on the left knee. Further investigation by performing MRI in July 2019 confirmed the left anterior cruciate ligament rupture (see Fig. 7 and 8). The patient underwent ACLR surgery using a peroneus longus tendon graft in August 2019, without any surgical complication during the follow-up.

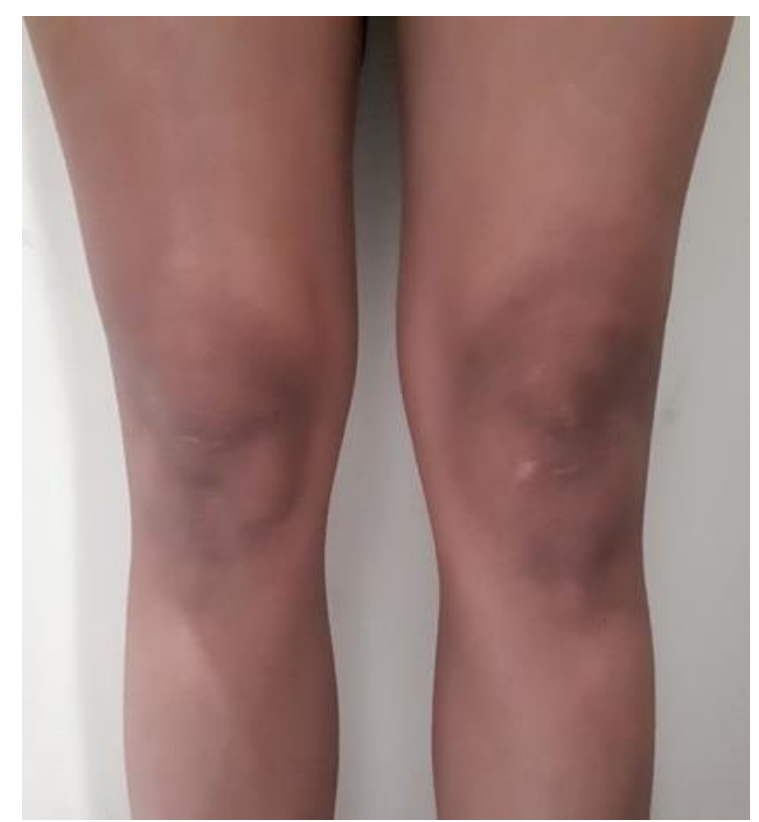

Fig. 7. Clinical presentation of the left ACL rupture from the fourth case.

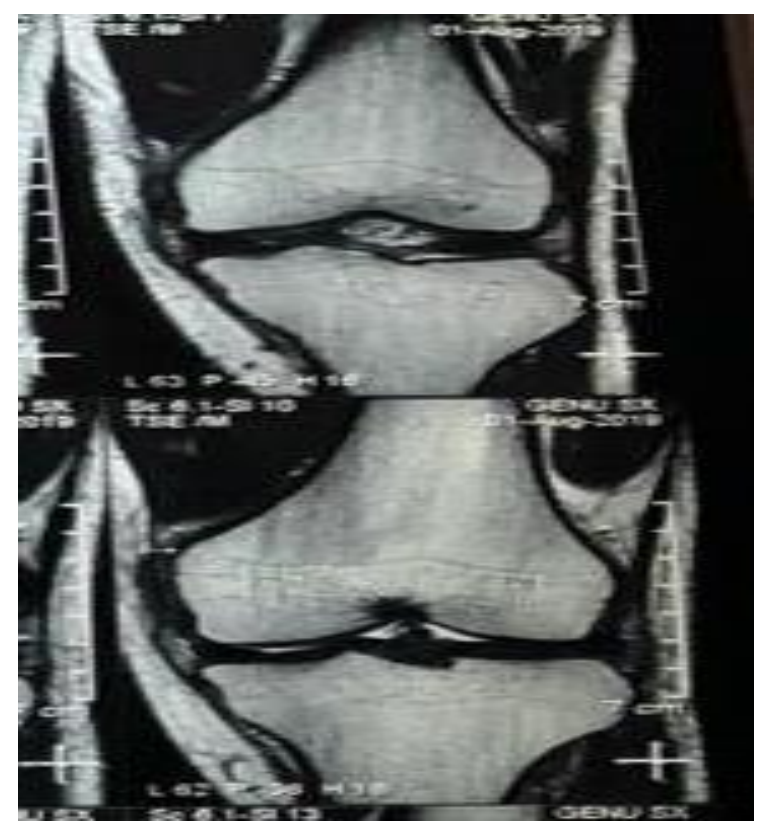

Fig. 8. MRI result showing the rupture of ACL. 


\section{E. Case 5}

The patient was a 50-year-old male with a chief complaint of a painful left knee. In the past three months prior to admission (March 2018), the patient fell from the stairs and bumped the left knee to the floor. The pain got better ever since; however, the patient experienced instability on his left knee when the patient walks upstairs or downstairs, and during high-intensity activity.

There was no swelling, deformity, or tenderness reported on the physical examination of the affected knee. The capillary refill time, dorsalis pedis artery, and pulse oximetry tests revealed normal results. Specific tests using Lachman Test and Anterior Drawer Test indicated the ruptured ACL on the left knee. The MRI was performed and the patient was diagnosed with left anterior cruciate ligament rupture in March 2018 (See Fig. 9 and 10). The ACL reconstruction using Peroneus Longus Tendon Graft was done in June 2018, without any surgical complication during the follow-up.

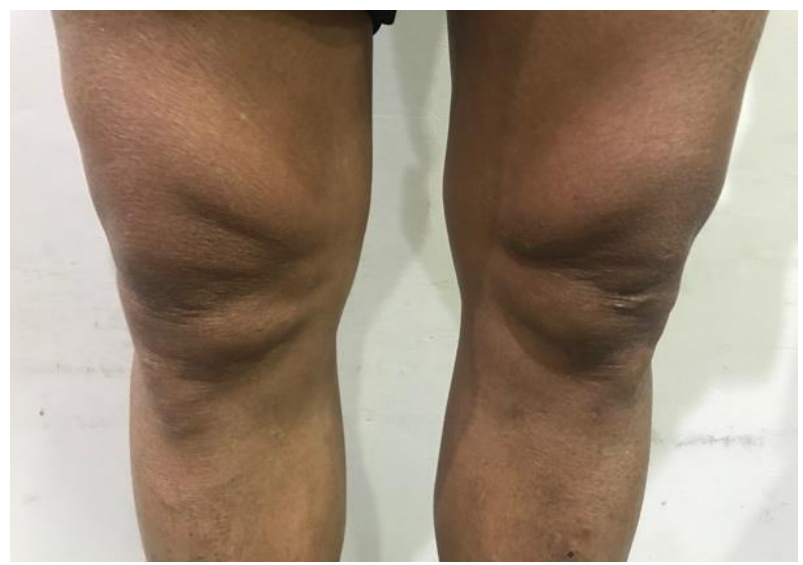

Fig. 9. Clinical presentation of the left ACL rupture from the fifth case.

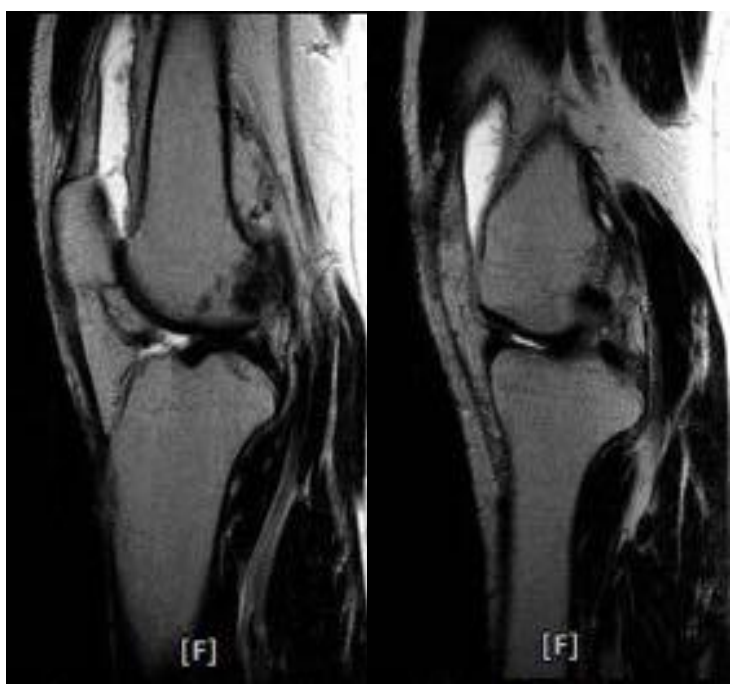

Figure. 10 The MRI presenting the ruptured ACL.

\section{F. Case 6}

A 21-year-old female came to the orthopedic outpatient clinic in Sanglah General Hospital, reported intermittent right knee pain and swelling four months before admission (January 2018). The pain was getting worse in the past two weeks with remarkable pain, especially during high-intensity activity. The patient had a history of a motorcycle accident, and the right knee was bumped to the ground in 2016.

The physical examination revealed swelling with no deformity. On palpation, tenderness was reported around the right knee; however, the capillary refill time, dorsalis pedis artery, and pulse oximetry test were within normal limit. The Lachman Test and Anterior Drawer Test were compromised due to pain in the right knee. Subsequent MRI investigation in April 2018 established the left anterior cruciate ligament rupture (see Fig. 11 and 12). The patient underwent an ACLR procedure using Peroneus Longus Tendon Graft in May 2018, without any surgical complication during the follow-up.

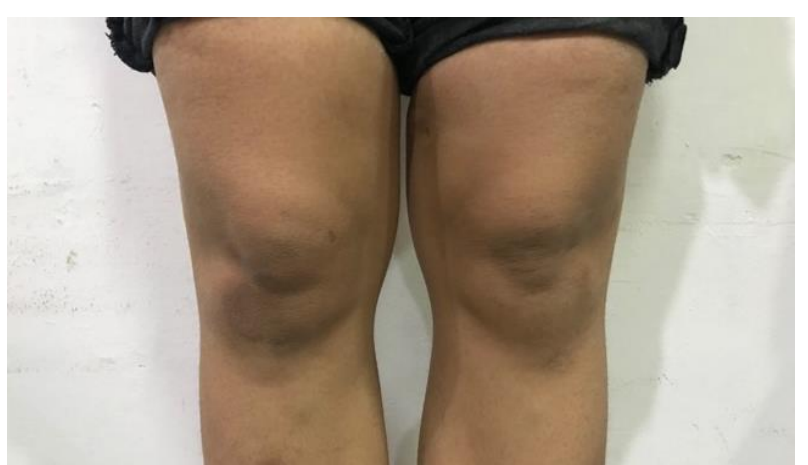

Fig. 11. Clinical features of the right ACL rupture from the sixth case.

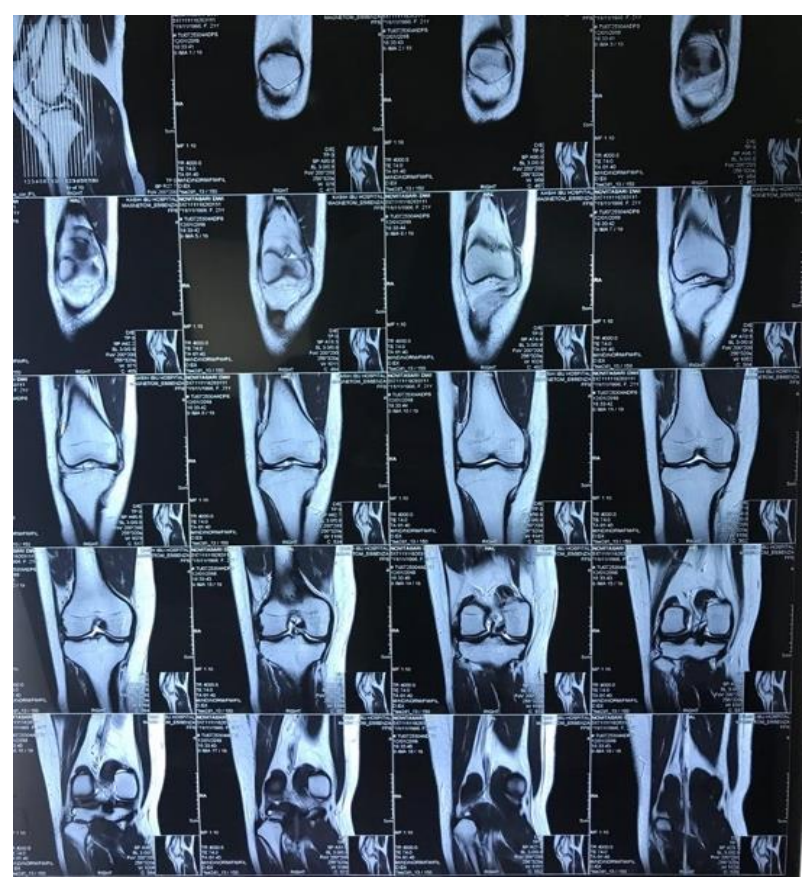

Fig. 12. A series of MRIs confirming the ruptured ACL.

\section{RESULTS}

We collected a case series of six consecutive patients with chronic ACL rupture, entailing the surgical management of ACL reconstruction using the peroneus longus tendon graft. The peroneus longus tendon graft is expected as an alternative choice of graft in ACL reconstruction. Therefore, the longterm evaluation of the surgical outcomes was measured to evaluate its effectiveness in knee and ankle functionality. All patients received a minimal one-year follow-up following the surgery. The assessment of post-operative functional outcomes of the knee was evaluated based on the IKDC (International Knee Documentation Committee) score. Meanwhile, the assessment of post-operative functional outcomes of the ankle was measured based on the AOFAS Score (The American Orthopaedic Foot and Ankle Society). 
The cases consisted of four male and two female patients, aged between 20-58 years old. The range follow-up was between 1-3 years. The overall functional outcome of IKDC and AOFAS scores is presented in Table I, showing satisfactory results [5].

TABLE I: IKDC AND AOFAS SCORING RESULTS FOLLOWING THE RECONSTRUCTION OF THE RUPTURED ACL USING PERONEUS LONGUS TENDON GRAFT

\begin{tabular}{cccccc}
\hline \hline No & Gender & Age (year) & $\begin{array}{c}\text { Follow-up } \\
\text { Onset (year) }\end{array}$ & IKDC & AOFAS \\
\hline 1. & Female & 39 & 3 & $67.8 \%$ & 82 \\
2. & Male & 58 & 3 & $67.8 \%$ & 77 \\
3. & Male & 26 & 3 & $78.1 \%$ & 95 \\
4. & Male & 20 & 1 & $68.9 \%$ & 73 \\
5. & Male & 52 & 2 & $75.8 \%$ & 92 \\
6. & Female & 23 & 2 & $86.2 \%$ & 95 \\
\hline \hline
\end{tabular}

IKDC, International Knee Documentation Committee; AOFAS, The American Orthopaedic Foot and Ankle Society.

\section{DISCUSSION}

Injuries to the anterior cruciate ligament (ACL) occur in the active general population and are particularly common in athletes performing in knee-pivoting sports. After an ACL injury, the knee has a significant risk of functional instability, meniscal tears, and subsequent osteoarthritis. Therefore, to restore knee stability and decrease the risk of subsequent injuries, ACL reconstruction (ACLR) is often performed. Excellent results have been widely reported for ACLR in the restoration of knee stability [5].

Despite the high reported success rates, ACLR is not to be taken lightly. Patients must invest a significant amount of time, discomfort, effort, and financial burden during the 6-12 months of the recovery period. In addition, the post-operative complication involving ACL graft re-tear after undergoing surgery and rehabilitation is a devastating event for the patient as well as the family, coach, therapist, and surgeon. This often results in a revision ACLR procedure requiring further ACL graft [6].

The ACL reconstruction, followed by both autografts or allografts, improves knee functionality and has already been studied extensively. Among these grafts, bone-patellar tendon-bone (BPTB) and four-strand hamstring autografts are the two most common autografts used, and each exhibits its advantages and disadvantages. The risks of patellar fracture, knee extension weakness, and anterior knee pain increase in using BPTB graft. Meanwhile, the hamstrings grafts often fall short in diameter and length, leading to postoperative hamstring weakness, so the knee is susceptible to unprotected anterior drawer force [4], [7].

Peroneus longus tendon autograft is an alternative graft choice and has been commonly used in several orthopedic practices, including spring ligament reconstruction, deltoid ligament reconstruction, and medial patellofemoral ligament (MPFL) reconstruction (anterior half of the peroneus longus tendon). The synergistic anatomical function of the peroneus longus and peroneus brevis supports the possibility of this procedure. Several studies revealed the role of peroneus brevis as a more effective evertor of the ankle, indicating the peroneus longus tendon graft harvest potential. In addition, previous studies reported satisfactory results after ACL reconstruction with the peroneus longus tendon in terms of both functional outcome and knee stability [8]-[10].

In this case series, we successfully treated six patients with chronic ACL rupture by performing ACLR using peroneus longus tendon graft. Intraoperatively, the operation was successful with no significant difficulties from the harvested peroneus longus tendon graft and ACL reconstruction with the graft. All patients presented with no post-operative complications, including local surgical site infection.

The rehabilitation procedure following ACLR was performed in the Rehabilitation Unit of Sanglah General Hospital. The functional outcomes follow-up using AOFAS and IKDC score were applied to the patient with the minimum one-year post-operative period and had completed the rehabilitation program.

The result of this case series is correspondent with the previous studies. We found that ACL reconstruction using peroneus longus tendon as the alternative graft showed a satisfactory functional outcome for knee stability and presented no negative outcome for functional outcome of the ankle, based on IKDC and AOFAS scores [11].

\section{CONCLUSION}

The case series of the six patients with chronic ACL ruptures concludes that the peroneus longus tendon graft is a suitable choice of graft in ACL reconstruction. In addition, the post-operative evaluation exhibits excellent safety and effectiveness based on the knee and ankle functional outcome using AOFAS and IKDC scoring.

\section{REFERENCES}

[1] S. Xiaoxiao, L. Qiangqiang, B. W. Zongfang, X Qian, D. Chen, Q. Jiang, "Predicting the graft diameter of the peroneus longus tendon for anterior cruciate ligament reconstruction," Medicine, vol. 97, pp.44, 2018.

[2] H. Xu, W. Lin, G. Jin, D. Xin, J. Z, K. Kang, J. Dong, S. Gao, B. Chen, "Graft choice for anatomic anterior cruciate ligament reconstruction The comparison between thin autograft and thick hybrid graft. An observational study," Medicine, vol. 98, pp. 37, 2018.

[3] C. Angthong, B. Chernchujit, A. Apivatgaroon, K. Chaijenkit, P. Nualon, K. Suchao-in, "The Anterior Cruciate Ligament Reconstruction with the Peroneus Longus Tendon: A Biomechanical and Clinical Evaluation of the Donor Ankle Morbidity," J Med Assoc Thai, vol. 98, pp. 6, 2015.

[4] S. Mohanty, A. Mohanty, A. Mishra, A. Mohanty, "Evaluation of results of peroneus longus graft in primary a.c.l reconstruction: a prospective study," International Journal of Scientific Research, vol. 7, pp. 9, 2019.

[5] N. G. Mohtadi, D. S. Chan, K. N. Dainty, D. B. Whelan, "Patellar tendon versus hamstring tendon autograft for anterior cruciate ligament rupture in adults," Medicine, vol. 7, pp. 9, 2011.

[6] S. Rhatomy, A. I. Z. Asikin, A. E. Wardani, T. Rukmoyo, I. Lumbangaol, N. C. Budhiparama, "Peroneus longus autograft can be recommended as a superior graft to hamstring tendon in single-bundle ACL reconstruction," European Society of Sports Traumatology, Knee Surgery, Arthroscopy, 2019.

[7] B. R. William, S. J. Ellis, T. W. Deyer, H. Pavlon, "Reconstruction of the spring ligament using a peroneus longus autograft tendon transfer," Foot Ankle Int, vol. 31, 2010.

[8] C. Xu, J. Zhao, G. Xie, "Medial patella-femoral ligament reconstruction using the anterior half of the peroneus longus tendon as a combined procedure for recurrent patellar instability," Asia Pac J Sport Med Arthrosc Rehabil Tachnol, vol. 4, 2016.

[9] C. K. Christopher, D. P. Angela, K. R. Emily, L. J. Huston, T. E. Hewett, K. P. Spindler, "Change in Anterior Cruciate Ligament Graft Choice and Outcomes Over Time," Arthroscopy, vol. 33, pp. 11, 2017.

[10] S. Fu-Dong, W. Xue-Chen, Z. Shao-Ping, D. E. Hess, Z. Jin-Zeng, L. Shi-Jie, Y. Zhang, M. Zhang-Guang, M. Xiao-Guang, C. Ze-Jun, L. 
Chang-Jiang, H. Wan-Ning, "Peroneus Longus Tendon Autograft is a Safe and Effective Alternative for Anterior Cruciate Ligament Reconstruction," The Journal of Knee Surgery, 2018.

[11] D. V. Ilchenko, A. V. Korolev, A. A. Kardanov, "Long-term functional results of first metatarsophalangeal joint arthrodesis for hallux rigidus," Geniji Orthopedic, vol. 26, pp. 1, 2020. 